\title{
Indocyanine Green Labels an Orthotopic Nude-Mouse Model of Very-Early Colon-Cancer Liver Metastases
}

\author{
YOSHIHIKO TASHIRO ${ }^{1,2,3}$, HANNAH M. HOLLANDSWORTH ${ }^{2,4}$, HIROTO NISHINO $^{1,2}$, JUN YAMAMOTO ${ }^{1,2}$, \\ SIAMAK AMIRFAKHRI ${ }^{2,4}$, FILEMONI FILEMONI ${ }^{2,4}$, NORIHIKO SUGISAWA $^{1,2}$, TAKESHI AOKI $^{3}$, \\ MASAHIKO MURAKAMI ${ }^{3}$, ROBERT M. HOFFMAN ${ }^{1,2,4}$ and MICHAEL BOUVET ${ }^{2,4}$ \\ ${ }^{1}$ AntiCancer Inc, San Diego, CA, U.S.A.; \\ ${ }^{2}$ Department of Surgery, University of California, San Diego, CA, U.S.A.; \\ ${ }^{3}$ Department of General and Gastroenterological Surgery, Showa University School of Medicine, Tokyo, Japan; \\ ${ }^{4}$ VA San Diego Healthcare System, San Diego, CA, U.S.A.
}

\begin{abstract}
Background/Aim: Near-infrared (NIR) fluorescence imaging using indocyanine green (ICG) is used to visualize colon-cancer liver metastases (CCLM) during surgery. The present study aimed to use ICG to visualize small CCLM in an orthotopic mouse model. Materials and Methods: A 3-mm fragment of HT29 human colon cancer was implanted to the liver of 5 athymic nude mice (nu/nu). The Pearl Trilogy Small Animal Fluorescence Imaging system was used $24 \mathrm{~h}$ after intravenous (IV) injection of $0.025 \mathrm{mg}(0.25 \mathrm{mg} / \mathrm{ml})$ ICG. Results: In four of the five mice, tumor fluorescence was detected. Small tumors (approximately $3 \mathrm{~mm}$ ) were distinctly visualized with a minimal fluorescence liver signal, with a mean tumor to liver signal ratio of 1.81 (SD \pm 0.167). Conclusion: Small CCLM can be clearly identified by ICG fluorescence in an orthotopic nude-mouse model. This model is translatable to the clinic for the detection of occult early CCLM.
\end{abstract}

Indocyanine green (ICG) fluorescence imaging is widely applied in various surgical fields. In liver surgery, ICG fluorescence imaging is used in tumor detection, tattooing, biliary-tract contrast, liver segmentation and determination of surgical margins (1-3). Laparoscopic liver resection has become popular in recent years; however, palpation and visualization of liver tumors is difficult in laparoscopic surgery. Therefore, the identification of liver tumors using

This article is freely accessible online.

Correspondence to: Michael Bouvet, MD, Department of Surgery, UCSD Moores Cancer Center, 3855 Health Sciences Drive \#0987, La Jolla, CA, 92093, U.S.A. Tel: +1 8588226191, Fax: +1 858822619, e-mail: mbouvet@health.ucsd.edu

Key Words: ICG, fluorescence, colon cancer, liver metastasis, detection, orthotopic mouse model.
ICG fluorescence signals is an extremely useful tool in laparoscopic liver surgery.

The most frequently used imaging modalities to assess liver tumors are computed tomography (CT) and intra-operative ultrasonography (IOUS). However, even with the combined use of these modalities, $6 \%$ to $20 \%$ of liver metastases are not identified (4-6). This may lead to inadequate assessment of the extent of disease and incomplete resection, which can have a significant impact on outcomes and prognosis (7). Ishizawa et al. have shown that primary hepatocellular carcinoma and colorectal-cancer liver metastases could be identified using near-infrared (NIR) fluorescence imaging (8). Currently, NIR fluorescence imaging is becoming more widely used in the operative setting for the identification of hepatic pathology and anatomy.

Fluorescence imaging with ICG of colon-cancer liver metastases (CCLM) in rat models has been previously established (9). The present study aimed to develop an orthotopic nude-mouse model for fluorescence visualization of early and small CCLM using ICG.

\section{Materials and Methods}

Animals. Athymic nu/nu male nude mice (8-10 weeks) were used in this study. Mice were housed in a barrier facility on a highefficacy particulate arrestance (HEPA)-filtered rack under standard conditions of 12-h light/dark cycles. Animal studies were performed under an AntiCancer Institutional Animal Care and Use Committee (IACUC)-protocol specifically approved for this study and in accordance with the principles and procedures outlined in the National Institutes of Health Guide for the Care and Use of Animals under Assurance Number A3873-1. All mouse surgical procedures and imaging were performed with the animals anesthetized by subcutaneous injection of a ketamine mixture $(0.02 \mathrm{ml}$ solution of $20 \mathrm{mg} / \mathrm{kg}$ ketamine, $15.2 \mathrm{mg} / \mathrm{kg}$ xylazine, and $0.48 \mathrm{mg} / \mathrm{kg}$ acepromazine maleate) (10).

Cell line. The human colon cancer cell line HT29 was obtained (ATCC, Manassas, VA, USA) and cultured in RPMI-1640 with 10\% 


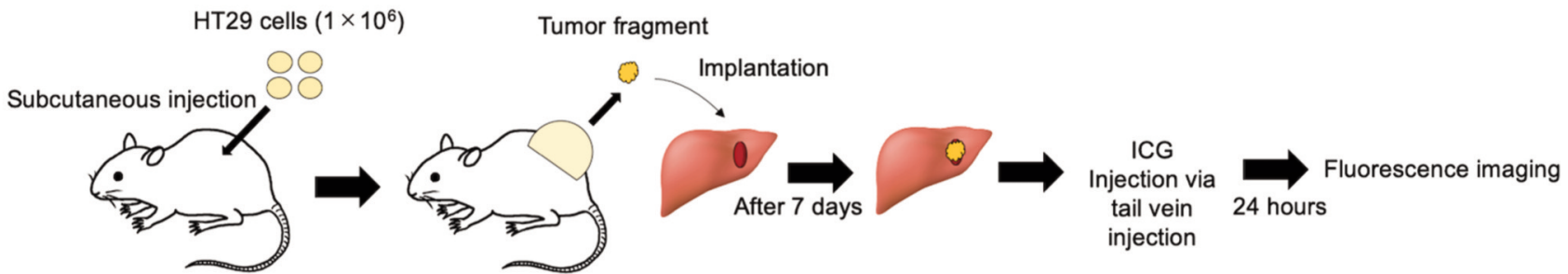

Figure 1. Experimental schema.
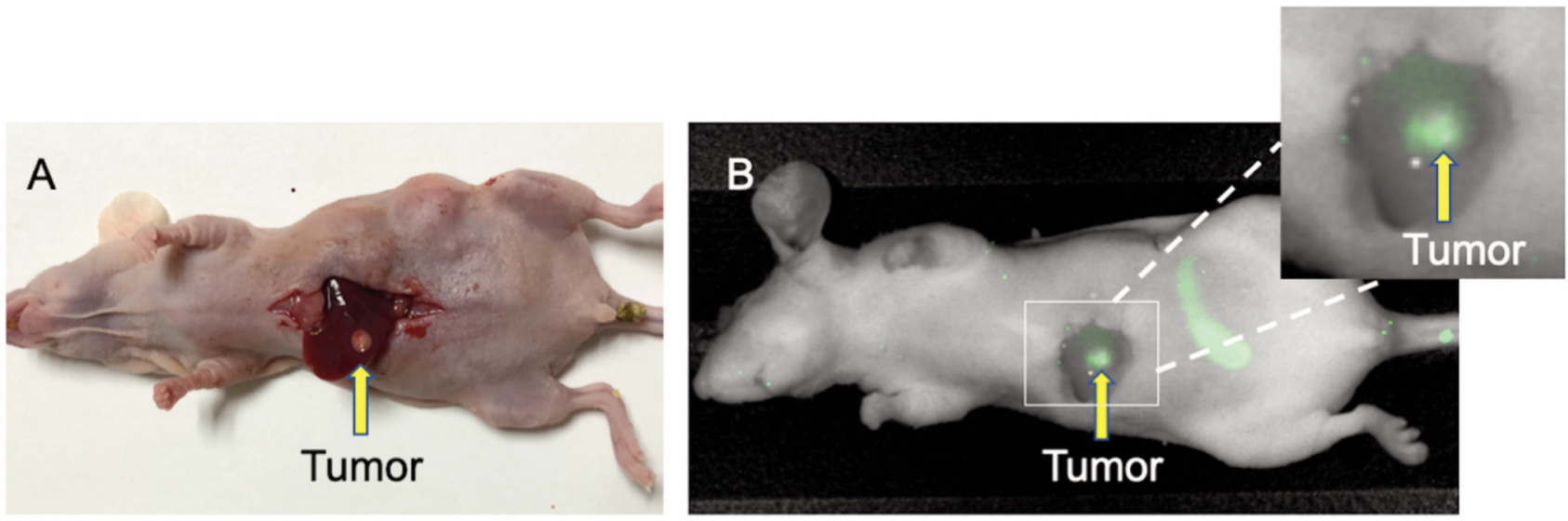

Figure 2. Indocyanine green (ICG) tumor fluorescence detected in a mouse model of CCLM. (A) Bright-light image of the colon-cancer livermetastases (CCLM) model. The tumor measured approximately $3 \mathrm{~mm}$ in diameter (B) Fluorescence image obtained 24 h after administration of $0.025 \mathrm{mg}$ ICG via tail-vein injection. Tumor margins are distinctly identified with minimal fluorescence liver signal.

fetal bovine serum and $1 \%$ penicillin-streptomycin mixture. Cells were cultured at $37^{\circ}$ in a humidified atmosphere containing $5 \% \mathrm{CO}_{2}$.

Study design. The CCLM model in nude mice has been previously established (11). Washed colon-cancer cells, exceeding 95\% cell viability by trypan blue dye exclusion were inoculated into nude mice. HT29 cells $\left(1 \times 10^{6}\right)$ were subcutaneously injected into the bilateral flanks. Once tumor growth reached $10 \mathrm{~mm}$ in diameter (in approximately 1-2 weeks), tumors were harvested and cut into small fragments. After anesthesia was administered, a $1 \mathrm{~cm}$ skin incision was made on the upper abdomen through the skin, fascia and peritoneum and the liver was exposed. A 5-mm incision was made in the left lobe of the liver, and a $3 \mathrm{~mm}$ tumor fragment was implanted into the liver. Fixation of the tumor was confirmed at 7 days post-implantation via midline laparotomy after the mouse was anesthetized (Figure 1).

Indocyanine green. ICG was purchased from Daiichi Sankyo, Tokyo, Japan. ICG was diluted to $2.5 \mathrm{mg} / \mathrm{ml}$ with an equipped solution and ten times with phosphate-buffered saline. Final ICG concentration was $0.25 \mathrm{mg} / \mathrm{ml}$.

Mouse imaging. For intra-vital imaging, the Pearl Trilogy Small Animal Fluorescence Imaging system was used (LI-COR, Lincoln,
NE, USA). The Pearl Trilogy is equipped for sensitive imaging of 700 and 800 nanometer NIR fluorophores. Images were obtained with standard imaging settings. One week after tumor implantation, mice were administered ICG via tail vein injection. Twenty-four hours later, laparotomy was performed and images were obtained with the Pearl Trilogy.

Statistical analysis. Imaging analysis was performed using Image Studio Software Small Animal Imaging Analysis (LI-COR, Lincoln, NE, USA). An elliptical area of interest was drawn over the tumor parenchyma on the software and set as the background. An area of interest was also drawn around the tumor margins. Tumor-to-liver ratio (TLR) was calculated for each mouse by dividing maximum tumor fluorescence by maximum liver fluorescence. Mean TLR was calculated using descriptive statistics on SPSS version 24.

\section{Results}

One week after implantation of the HT29 tumor fragment, laparotomy demonstrated adequate tumor growth in the liver (Figure 2A). Intra-vital fluorescence imaging $24 \mathrm{~h}$ after administration of ICG demonstrated that CCLM was 
detected in 4 out of 5 mice (Figure 2B). Imaging also demonstrated minimal background liver fluorescence signal with a mean $\mathrm{TLR}=1.81$ ( $\mathrm{SD} \pm 0.167)$. Tumors approximately $3 \mathrm{~mm}$ in diameter were readily visualized (Figure 2B). The fluorescence over the abdomen seen in Figure 2B was due to cecal fluorescence enhancement due to delivery of ICG to this well-perfused area.

\section{Discussion}

This study serves as a proof of concept that orthotopic mouse models are an ideal alternative to more costly models for further pre-clinical evaluation of ICG for CCLM. Although CCLM ICG fluorescence imaging in rats has been previously established (9), there are no studies to date using orthotopic mouse models for CCLM fluorescence imaging with ICG.

With the transition to minimally-invasive techniques, detection of CCLM has become difficult. Current techniques for intra-operative detection of liver metastases include ultrasound. Previous studies have demonstrated that occult tumors not detected by preoperative imaging and intra-operative ultrasound were able to be detected with intra-operative fluorescence imaging (12, 13). Further studies in orthotopic mouse models of CCLM need to be performed to optimize the concentration and timing of imaging, for improved translatability to clinical studies.

In the present study, we demonstrated that tumor fluorescence could be detected in $80 \%$ of mice $24 \mathrm{~h}$ after ICG injection in an orthotopic mouse model of CCLM. In addition, this study is promising for the early detection of small CCLM with ICG. In summary, orthotopic mouse models are feasible for fluorescence imaging of CCLM using ICG and are clinically relevant (14-19).

\section{Conflicts of Interest}

There are no financial or other interests regarding the submitted manuscript that might be construed as a conflict of interest.

\section{Authors' Contributions}

YT and MB designed the experiments. YT, HN, HMH and JY performed experiments, analyzed data and wrote the paper; TA, $\mathrm{MM}, \mathrm{HMH}, \mathrm{SA}, \mathrm{FF}$ and RMH gave technical support and conceptual advice. All Authors were involved in editing and approval of the final manuscript.

\section{Acknowledgements}

This study was funded by VA Merit Review grant numbers 1 I01 BX003856-01A1 and 1 I01 BX004494-01 (MB) and NIH/NCI T32CA121938 (HH).

\section{References}

1 Aoki T, Murakami M, Koizumi T, Matsuda K, Fujimori A, Kusano T, Enami Y, Goto S, Watanabe M and Otsuka K: Determination of the surgical margin in laparoscopic liver resections using infrared indocyanine green fluorescence. Langenbecks Arch Surg 403: 671-680, 2018. PMID: 29915961. DOI: $10.1007 / \mathrm{s} 00423-018-1685-\mathrm{y}$

2 Aoki T, Yasuda D, Shimizu Y, Odaira M, Niiya T, Kusano T, Mitamura K, Hayashi K, Murai N, Koizumi T, Kato H, Enami Y, Miwa $\mathrm{M}$ and Kusano M: Image-guided liver mapping using fluorescence navigation system with indocyanine green for anatomical hepatic resection. World J Surg 32: 1763-1767, 2008. PMID: 18543027. DOI: 10.1007/s00268-008-9620-y

3 Ishizawa T, Tamura S, Masuda K, Aoki T, Hasegawa K, Imamura $\mathrm{H}$, Beck Y and Kokudo N: Intraoperative fluorescent cholangiography using indocyanine green: a biliary road map for safe surgery. J Am Coll Surg 208: e1-4, 2009. PMID: 19228492. DOI: 10.1016/j.jamcollsurg.2008.09.024

4 Leen E, Ceccotti P, Moug SJ, Glen P, MacQuarrie J, Angerson WJ, Albrecht T, Hochmann J, Oldenburg A, Ritz JP and Horgan PG: Potential value of contrast-enhanced intraoperative ultrasonography during partial hepatectomy for metastases: an essential investigation before resection? Ann Surg 243: 236-240, 2006. PMID: 16432357. DOI: 10.1097/01.sla.0000197708. 77063.07.

5 Sahani DV, Kalva SP, Tanabe KK, Hayat SM, O'Neill MJ, Halpern EF, Saini $S$ and Mueller PR: Intraoperative US in patients undergoing surgery for liver neoplasms: comparison with MR imaging. Radiology 232: 810-814, 2004. PMID: 15273336. DOI: $10.1148 /$ radiol.2323030896

6 Nomura K, Kadoya M, Ueda K, Fujinaga Y, Miwa S and Miyagawa S: Detection of hepatic metastases from colorectal carcinoma: comparison of histopathologic features of anatomically resected liver with results of preoperative imaging. J Clin Gastroenterol 41: 789-795, 2007. PMID: 17700429. DOI: 10.1097/01.mcg.0000225676.22218.08

7 Hazhirkarzar B, Khoshpouri P, Shanghaghi, Ghasabeh MA, Pawlik TM and Kamel IR: Current state of the art imaging approaches for colorectal liver metastases. HepatoBiliary Surg Nutr 9: 35-48, 2020. PMID: 32140477. DOI: 10.21037/hbsn. 2019.05.11

8 Ishizawa T, Fukushima N, Shibahara J, Masuda K, Tamura S, Aoki T, Hasegawa K, Beck Y, Fukayama M and Kokudo N: Real-time identification of liver cancers by using indocyanine green fluorescent imaging. Cancer 115: 2491-2504, 2009. PMID: 19326450. DOI: $10.1002 /$ cncr.24291.

9 Van der Vorst JR, Hutteman M, Mieog JS, de Rooji KE, Kaijzel EL, Lowik CWGM, Putter H, Kuppen PJK, Frangioni JV, van de Velde CJH and Vehrmeijer A: Near-infrared fluorescence imaging of liver metastases in rats using indocyanine green. J Surg Res 174: 266-271, 2011. PMID: 21396660. DOI: 10.1016/ j.jss.2011.01.009

10 Yang M, Reynoso J, Bouvet M and Hoffman RM: A transgenic red fluorescent protein-expressing nude mouse for color-coded imaging of the tumor microenvironment. J Cell Biochem 106: 279-284, 2009. PMID: 19097136. DOI: 10.1002/jcb.21999

11 Oshiro H, Tome Y, Kiyuna T, Yoon SN, Lwin TM, Han Q, Tan Y, Miyake K, Higuchi T, Sugisawa N, Katsuya Y, Park JH, Zang Z, Razmjooei S, Bouvet M, Clary B, Singh SR, Kanaya F, Nishida 
K and Hoffman RM: Oral recombinant methioninase overcomes colorectal-cancer liver metastasis resistance to the combination of 5-Fluorouracil and oxaliplatinum in a patient-derived orthotopic xenograft mouse model. Anticancer Res 39: 4667-4671, 2019. PMID: 31519565. DOI: 10.21873/anticanres.13648

12 Gotoh K, Yamada T, Ishikawa O, Takahashi H, Eguchi H, Yano M, Ohigashi H, Tomita Y, Miyamoto Y and Imaoka S: A novel image-guided surgery of hepatocellular carcinoma by indocyanine green fluorescence imaging navigation. J Surg Oncol 100: 75-79, 2009. PMID: 19301311. DOI: $10.1002 /$ jso.21272

13 Morita Y, Sakaguchi T, Unno N, Shibasaki Y, Suzuki A, Fukumoto K, Inaba K, Baba S, Takehara Y, Suzuki S and Konno $\mathrm{H}$ : Detection of hepatocellular carcinomas with near-infrared fluorescence imaging using indocyanine green: its usefulness and limitation. Int $\mathrm{J}$ Clin Oncol 18: 232-241, 2013. PMID: 22200990. DOI: $10.1007 / \mathrm{s} 10147-011-0367-3$

14 Hoffman RM: Orthotopic metastatic mouse models for anticancer drug discovery and evaluation: A bridge to the clinic. Invest New Drugs 17: 343-359, 1999. PMID: 10759402. DOI: 10.1023/a:1006326203858

15 Fu XY, Besterman JM, Monosov A and Hoffman RM: Models of human metastatic colon cancer in nude mice orthotopically constructed by using histologically intact patient specimens. Proc Natl Acad Sci U S A 88: 9345-9349, 1991. PMID: 1924398. DOI: $10.1073 /$ pnas.88.20.9345
16 Hoffman RM: Patient-derived orthotopic xenografts: better mimic of metastasis than subcutaneous xenografts. Nat Rev Cancer 15: 451-452, 2015. PMID: 26422835. DOI: $10.1038 / \mathrm{nrc} 3972$

17 Kuo TH, Kubota T, Watanabe M, Furukawa T, Teramoto T, Ishibiki K, Kitajima M, Moossa AR, Penman S and Hoffman RM: Liver colonization competence governs colon cancer metastasis. Proc Natl Acad Sci U S A 92: 12085-12089, 1995. PMID: 8618849. DOI: 10.1073/pnas.92.26.12085

18 Hoffman RM: Orthotopic is orthodox: Why are orthotopictransplant metastatic models different from all other models? J Cell Biochem 56: 1-3, 1994. PMID: 7806583. DOI: $10.1002 /$ jcb. 240560102

19 Patient-Derived Mouse Models of Cancer. Patient-Derived Orthotopic Xenografts (PDOX). Hoffman RM (eds.). New York, Humana Press, 2017. DOI: 10.1007/978-3-319-57424-0

Received May 5, 2020

Revised May 30, 2020

Accepted May 31, 2020 